\title{
Collective Ion Dynamics in Liquid Zinc: Evidence for Complex Dynamics in a Non-Free-Electron Liquid Metal
}

\author{
M. Zanatta, ${ }^{1,2,{ }^{*}}$ F. Sacchetti, ${ }^{1,2}$ E. Guarini, ${ }^{3}$ A. Orecchini, ${ }^{1}$ A. Paciaroni, ${ }^{1}$ L. Sani, ${ }^{1}$ and C. Petrillo ${ }^{1,2}$ \\ ${ }^{1}$ Dipartimento di Fisica e Geologia, Università di Perugia, Via Alessandro Pascoli, I-06123 Perugia, Italy \\ ${ }^{2}$ Istituto Officina Materiali, Unità di Perugia, clo Dipartimento di Fisica e Geologia, Università di Perugia, \\ Via Alessandro Pascoli, I-06123 Perugia, Italy \\ ${ }^{3}$ Dipartimento di Fisica e Astronomia, Università di Firenze, via G. Sansone 1, I-50019 Sesto Fiorentino, Italy
}

(Received 1 October 2014; published 4 May 2015)

\begin{abstract}
A detailed inelastic neutron scattering investigation of the $\mathrm{THz}$ dynamics of liquid zinc is presented. The observed $Q$ dependence clearly reveals the existence of a complex dynamics made up of two distinct excitations. The highest energy mode is the prolongation of the longitudinal acoustic density fluctuations whereas the comparison with the phonon dynamics of crystalline hep zinc suggests a transverse acousticlike nature for the second one. This mode seems related to peculiar anisotropic interactions, possibly connected to the behavior of the crystalline phase.
\end{abstract}

DOI: 10.1103/PhysRevLett.114.187801

PACS numbers: 61.25.Mv, 61.05.F-, 62.60.+v

Since the seminal work of Copley and Rowe [1], who in 1974 demonstrated the existence of a long-living collective mode in liquid $\mathrm{Rb}$, the $\mathrm{THz}$ dynamics of a large number of liquid metals has been investigated by means of inelastic neutron and $x$-ray scattering (INS and IXS), e.g., Refs. [2-4]. The key observable in these studies is the dynamic structure factor $S(Q, \omega)$. Experiments provide a large body of information spanning from alkali metals and their alloys to polyvalent and transition metals and covering a wide range of thermodynamic states. Data seem universally characterized by the existence of a longitudinal acoustic mode that shows an almost well-defined sinusoidal dispersion over a wide range of momentum transfers $Q$, typically up to half the position $Q_{p}$ of the first maximum of the static structure factor. Several theoretical approaches have been proposed to describe the $\mathrm{THz}$ dynamics of liquid metals [5-10] but, even if in the case of alkali metals, alkali earths and polyvalent metals experimental data can be cast into a common trend [3], a consistent interpretation is not well defined yet [11].

As compared to that of liquid metals, the dynamics of nonmetallic liquids, like water, shows a more complex behavior and the $S(Q, \omega)$ is characterized by the existence of at least two different modes, e.g., Refs. $[12,13]$. These features arise in the microscopic region, well beyond the hydrodynamic regime, and are probably due to anisotropic interactions. Interestingly, recent studies point out the existence of a similar behavior also in liquid metals [14-18]. This is the case of liquid gallium, where IXS experiments [14,15] and molecular dynamics simulations [17] show the existence of a second low-energy mode that is identified as transverse acousticlike. The origin of this excitation might be related to anisotropic interactions originating peculiar structural arrangement such as transient cages [14] or $\mathrm{Ga}_{2}$ dimers [15].
In this Letter we present an inelastic neutron scattering study of the $\mathrm{THz}$ dynamics of liquid zinc. In the periodic table, zinc lies at the end of the transition metals series where the full $d$-like band still contributes to the screening of the electron-ion potential. In the crystalline phase, $\mathrm{Zn}$ has an hep structure with a room-temperature $c / a$ ratio of 1.856 [19], $\sim 14 \%$ higher than the ideal ratio $c / a=\sqrt{8 / 3} \simeq 1.633$. This rather anomalous value increases with temperature and indicates that specific interactions along the $c$ axis should be present to stabilize the hcp structure against the more isotropic fcc one [20]. This anisotropy could be also related to the anomalous electronic-topological transition observed at high pressure [20] and it makes liquid zinc a good candidate for displaying a complex dynamics. From the experimental point of view, $\mathrm{Zn}$ is an almost coherent neutron scatterer with a melting temperature $T_{m}=693 \mathrm{~K}$. In the hydrodynamic limit, the longitudinal sound velocity at the melting point is $v_{L}^{0}=2850 \mathrm{~m} / \mathrm{s}$ [21]. This fairly high value is well within the range of the time-of-flight neutron Brillouin spectrometer BRISP [22-24], installed at the Institut Laue Langevin research reactor (Grenoble, France) and properly designed to perform INS experiments at small momentum transfer $Q$. Experimental results reveal a complex dynamics, showing the existence of two distinct modes. A comparison with crystalline zinc phonons suggests an acousticlike character for both modes.

The sample was a 99.9\% pure ingot (Sigma, Aldrich) with natural isotopic composition, which was manipulated, weighted, and sealed into the sample container under the inert atmosphere of a He-filled glovebox. The empty cell was a slab-shaped $\mathrm{Nb}$ container with $0.5 \mathrm{~mm}$ thick windows, which provides both a relatively small scattering and a negligible mixing with liquid $\mathrm{Zn}$. The experiment was carried out with an incoming neutron wavelength of 


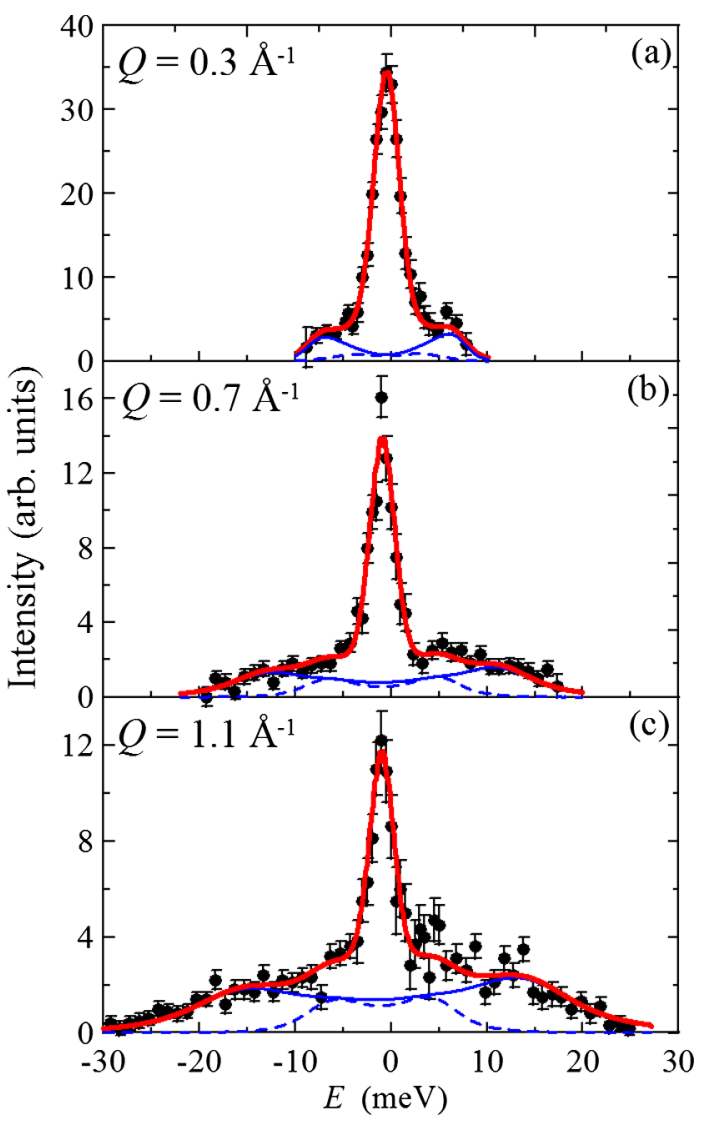

FIG. 1 (color online). Experimental data (black dots) at three typical $Q$ values, $Q=0.3 \AA^{-1}$ (a), $0.7 \AA^{-1}$ (b), and $1.1 \AA^{-1}$ (c). The thick red line represents the best fit to the data using the interacting modes model described in the text. Blue lines are the two inelastic components of the fit, $j=1$ (solid blue line) and $j=2$ (dashed blue line).

$0.98 \AA$ A. Measurements were performed in a standard furnace at $T_{\text {exp }}=773 \mathrm{~K}$. The measured sample intensity was reduced by carefully subtracting the contribution of the empty cell, the environment background, and multiple scattering, estimated using ad hoc Monte Carlo simulations. The instrument elastic resolution $R(\omega)$, measured using the incoherent scattering from a vanadium standard, was Gaussian shaped with a full width at half maximum (FWHM) of $2.8 \mathrm{meV}$.

The reduced scattering intensity $I(Q, \omega)$ is shown in Fig. 1 at some typical wave vector $Q$. Spectra are characterized by a resolution limited quasielastic peak with inelastic side structures that are related to the collective dynamics of the system and become more evident at small $Q$ values. The single scattering intensity can be written as

$$
I(Q, \omega)=A\left[R(\omega) \otimes \frac{k}{k_{0}} S(Q, \omega)\right],
$$

where $k_{0}$ and $k$ are the initial and the final neutron wave vector, respectively, and $A$ is a normalization constant.
Accurate simulation studies of $S(Q, \omega)$ of several liquids of various types have shown [11] that their longitudinal translational dynamics is well described by a model containing a term accounting for the propagation of the acoustic excitation and two quasielastic modes related to relaxation mechanisms building up the central peak of the spectrum. The latter are often difficult to disentangle from each other and from the resolution broadening when fitting the model to actual experimental data. Considering the broad experimental resolution, a simple empiric approach consists in modeling the $S(Q, \omega)$ with the sum of a Lorentzian for the quasielastic contribution and a damped harmonic oscillator (DHO) for the inelastic coherent one. This simple model has been widely exploited to describe the dynamics of several liquid metals [3], and it provides a reasonable good fit to the experimental spectra also in the case of liquid $\mathrm{Zn}$. However, the resulting dispersion curve, in the inset of Fig. 2(a), appears rather anomalous and strongly differs from the sinusoidal shape expected for a longitudinal acoustic mode, as it shows a plateau between 0.5 and $1.1 \AA^{-1}$.

In view of the findings of Refs. [14-18], this behavior could be ascribed to the presence of an additional mode having a $Q$-dependent intensity so that the single DHO appears shifted and broadened. However, since scattering experiments detect only longitudinal density fluctuations and modes characterized by the same symmetry interact with each other, the most general description of a system with many modes has to consider also their coupling. Consequently, the inelastic part of the spectrum is not the sum of two DHOs and the $S(Q, \omega)$ is described by the sum of a Lorentzian plus the dynamic structure factor of a system of two interacting oscillators having energies $\hbar \omega_{j}(Q),(j=1,2)$. This model was already successfully employed in amorphous $\mathrm{SiSe}_{2}$ where a complex dynamics is quite evident [25]. The Hamiltonian of the system is assumed to be

$\mathcal{H}=\mathcal{H}_{o}+\frac{1}{2} \sum_{\mathbf{Q}} U(\mathbf{Q})\left[\mathbb{Q}_{\mathbf{Q} 1}^{\dagger} \mathbb{Q}_{\mathbf{Q} 2}+\mathbb{Q}_{\mathbf{Q} 2}^{\dagger} \mathbb{Q}_{\mathbf{Q} 1}\right]+\mathcal{H}_{a}$,

where $\mathcal{H}_{o}=\frac{1}{2} \sum_{\mathbf{Q}_{j}}\left[\mathbb{P}_{Q j}^{\dagger} \mathbb{P}_{Q_{j}}+\omega_{j}^{2}(Q) \mathbb{Q}_{Q j}^{\dagger} \mathbb{Q}_{Q j}\right]$. Operators $\mathbb{P}_{Q j}$ and $\mathbb{Q}_{Q j}$ are the momentum and coordinate of the $j$ th oscillator, $U(\mathbf{Q})$ is the coupling parameter between the modes, and $\mathcal{H}_{a}$ contains all the higher order components. Following Refs. [7,26], the diagonal displacementdisplacement Green's function turns out to be

$$
G_{j j}(Q, \omega)=\frac{1}{2 \pi} \frac{\chi_{j}(Q, \omega)}{1-\chi_{1}(Q, \omega) \chi_{2}(Q, \omega)|U(Q)|^{2}},
$$

where the single-mode Green's function is given by $\chi_{j}(Q, \omega)=\left[\omega^{2}-\omega_{j}^{2}(Q)-\Sigma_{j}(Q, \omega)\right]^{-1}$. The self-energy $\Sigma_{j}(Q, \omega)$ is written as $i \omega \Gamma_{j}(Q)$, where $\Gamma_{j}(Q)$ is the 


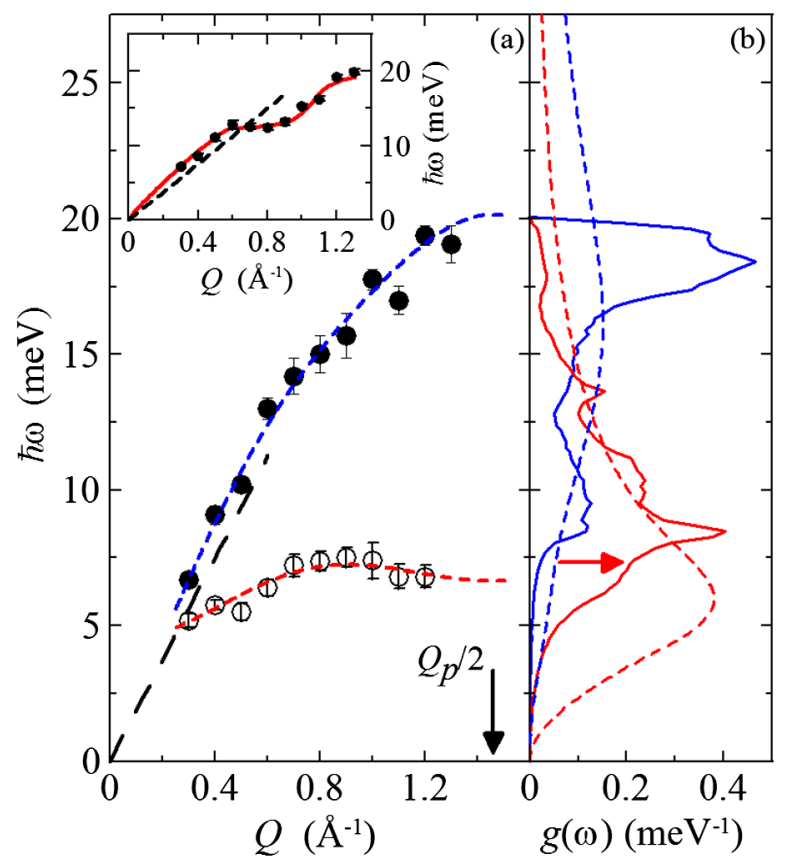

FIG. 2 (color online). (a) Dispersion curves for the bare energies $\hbar \omega_{1}(Q)$ (black dots) and $\hbar \omega_{2}(Q)$ (open black circles). The dashed blue line and the red one are guides to the eye marking the behavior of $\omega_{1}$ and $\omega_{2}$, respectively. The long dashed black line is the high frequency extrapolation of the longitudinal sound velocity measured in the hydrodynamic regime, $v_{L}^{0}=$ $2850 \mathrm{~m} / \mathrm{s}$ [21]. The black arrow indicates the boundary of the pseudo-Brillouin zone at $Q_{p} / 2=1.46 \AA^{-1}$. The dispersion curve obtained by using the single DHO model is reported in the inset (black dots) where the red line is a guide to the eye. (b) Vibrational density of states of crystalline hcp zinc of the longitudinal (blue line) and transverse (red line) modes, $g_{L}(\omega)$ and $g_{T}(\omega)$, respectively (see text). The red arrow marks the maximum of the $g(\omega)$ for purely transverse acoustic modes. The vibrational density of states calculated for $\omega_{1}(Q)$ and $\omega_{2}(Q)$, $g_{1}(\omega)$ (dashed blue line), and $g_{2}(\omega)$ (dashed red line), are also reported.

damping of the $j$ th mode. The coupling can be described by a Gaussian function $U(\mathbf{Q})=U_{0} Q \exp \left[-\left\langle R^{2}\right\rangle Q^{2} / 6\right],\left\langle R^{2}\right\rangle$ being the mean square range of the interaction in real space. The $S(Q, \omega)$ is the sum of the imaginary parts of the Green's functions $G_{11}(Q, \omega)$ and $G_{22}(Q, \omega)$, properly weighted by the unknown mode structure factors times $n(\omega)+1$, where $n(\omega)$ is the Bose factor [7,26]. Consistently, if $U(Q)=0$ each mode is described by a simple DHO. The resulting best-fit curves are shown in Fig. 1 by red lines together with the individual inelastic components (blue lines). The fit shows that $U(Q)$ is systematically nonzero in the whole $Q$ range. The model provides an accurate description of the spectral shapes and the so-obtained fit was also validated by performing a Bayesian analysis [27]. Figure 2(a) shows the dispersion relation of the two bare energies $\hbar \omega_{1}(Q)$ and $\hbar \omega_{2}(Q)$, as obtained from the fitting procedure.
The high-energy mode $\omega_{1}(Q)$ (black dots) displays a sinusoidal shape with a maximum at $Q_{p} / 2=1.46 \AA^{-1}$ (dashed blue line) and can be identified as a longitudinal acoustic mode. The apparent high-frequency sound velocity is $v_{L}^{\infty}=3380 \pm 60 \mathrm{~m} / \mathrm{s}$, about $20 \%$ higher than the hydrodynamic value $v_{L}^{0}$. Although $\mathrm{Zn}$ is not the best candidate for an approximation based on the homogeneous electron gas, we can compare the longitudinal mode dispersion relation to that derived by means of the Bohm-Staver approximation [4]. Assuming two electrons per atom, the sound velocity turns out to be $c_{\mathrm{BS}}=$ $4175 \mathrm{~m} / \mathrm{s}$. Conversely, if $e-e$ interactions are taken into account by using the compressibility sum rule for the homogeneous electron gas [28], we get $c_{\mathrm{ex}}=3195 \mathrm{~m} / \mathrm{s}$, which provides a fair agreement with the experimental value of $v_{L}^{\infty}$. A similar behavior was also observed in liquid mercury [29], which belongs to the same element group although in both cases the agreement might be accidental [4].

The low-lying mode $\omega_{2}(Q)$ (open black circles) shows an almost $Q$-independent behavior (dashed red lines) and its identification is not straightforward. As a matter of fact, the system isotropy prevents the precise knowledge of both vibrational eigenvalues and eigenvectors, which is therefore possible only in single crystals. To provide new insights on the nature of the modes in the liquid state, we can compare their behavior with the phonon dynamics of the crystalline phase. Phonon eigenvalues and eigenvectors of crystalline hcp zinc were calculated within the model of Ref. [30], using the force constants deduced in Ref. [31]. In particular, we can consider the vibrational density of states $g(\omega)$ of crystalline zinc in a hypothetical high temperature phase at $T_{\exp }>T_{m}$. The hop lattice parameters $a$ and $c$ have a smooth $T$ behavior up to the melting temperature so that they can be extrapolated to $T_{\text {exp }}$. To achieve the same density as the liquid phase, they were further expanded, keeping the $c / a$ ratio constant. Force constants were also uniformly reduced to match the longitudinal sound velocity with $v_{L}^{\infty}$. Phonon energies were thus calculated on 98304 points in the $1 / 24$ irreducible wedge of the Brillouin zone and then summed with appropriate weights to obtain the $g(\omega)$. This corresponds to the spherical average of the phonon dynamics over all $\mathbf{Q}$ values; hence, the so-obtained vibrational density of states is that of an ideal polycrystal. Moreover, the knowledge of the eigenvectors allows the projection of $g(\omega)$, either acoustic or optic, along the longitudinal $(L)$ and transverse (T) directions. The so-obtained $g_{L}(\omega)$ and $g_{T}(\omega)$ are thus reported in Fig. 2(b) and compared with the experimental vibrational density of states of $\omega_{1}(Q)$ and $\omega_{2}(Q), g_{1}(\omega)$ and $g_{2}(\omega)$, respectively. Even if softened and broadened by the solid-liquid transition, the peaks in $g_{1}(\omega)$ and $g_{2}(\omega)$ correspond to the main features of $g_{L}(\omega)$ and $g_{T}(\omega)$. This supports the longitudinal acoustic nature of $\omega_{1}(Q)$, already suggested by the dispersion curve. On the other hand, the 


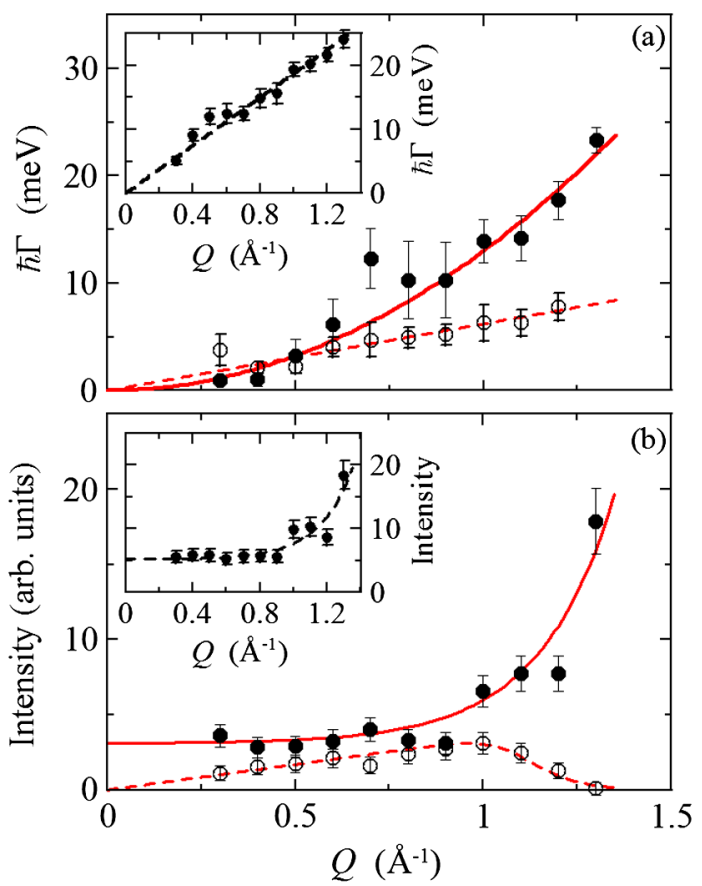

FIG. 3 (color online). (a) Damping parameters $\Gamma_{1}$ (black dots) and $\Gamma_{2}$ (open black circles). The red lines represent a quadratic trend (solid line) and a linear one (dashed line). The damping obtained within the single DHO model is reported in the inset with a linear fit (dashed line). (b) Intensity of $\omega_{1}(Q)$ (black dots) and $\omega_{2}(Q)$ (open black circles). The red lines are a guide to the eye. The intensity obtained by using the single DHO model is reported in the inset where the dashed line is a guide to the eye.

low-lying mode seems related to transverse phonons, and, in particular, to the acoustic part of the $g_{T}(\omega)$, whose peak, marked by the red arrow in Fig. 2(b), is very close to that of $g_{2}(\omega)$.

Figure 3(a) shows the damping parameter $\Gamma(Q)$. When a single DHO is used (see inset), $\Gamma(Q)$ shows a linear $Q$ trend, which is typical of other liquid metals analyzed with the same model [1-4]. Conversely, the model with interacting modes displays a different behavior. In particular, the damping of the low-lying mode shows a linear $Q$ dependence, while the longitudinal one shows a quadratic trend. This result is rather new and compares well with nonmetallic systems, e.g., Refs. [32-34].

Further information is obtained from the integrated intensity of the two modes, obtained by the fitting procedure. The results are reported in Fig. 3(b). The longitudinal mode shows an almost constant trend up to the region where it increases approaching the position of the first diffraction peak, $Q_{p}=2.93 \AA^{-1}$ [35]. This behavior is expected from general arguments [7]. Interestingly, the low-lying mode shows a completely different trend as its intensity is quite small at low momentum, decreasing again when $Q_{p} / 2$ is approached.

The existence of the transverse acousticlike mode $\omega_{2}(Q)$ might be related to anisotropic interactions of the $\mathrm{Zn}$ potential and possibly connected also to the existence of peculiar structural units such as dimers even in the liquid phase. Of course, when the probed length scale is increased, i.e., $Q$ is decreased, the microscopic structure becomes irrelevant. In this limit, coherently with a transverse acoustic nature, the intensity of the low-energy mode tends to zero and it eventually vanishes in the hydrodynamic region.

The Institut Laue Langevin (Grenoble, France) is gratefully acknowledged for allocation of beam time at the BRISP spectrometer.

*marco.zanatta@fisica.unipg.it

[1] J. R. D. Copley and J. M. Rowe, Phys. Rev. Lett. 32, 49 (1974).

[2] T. Scopigno, G. Ruocco, and F. Sette, Rev. Mod. Phys. 77, 881 (2005).

[3] L. E. Bove, C. Petrillo, and F. Sacchetti, Condens. Matter Phys. 11, 119 (2008).

[4] L. Sani, C. Petrillo, and F. Sacchetti, Phys. Rev. B 90, 024207 (2014).

[5] H. Mori, Prog. Theor. Phys. 33, 423 (1965); 34, 399 (1965).

[6] D. Pines and P. Nozieres, The Theory of Quantum Liquids (W. A. Benjamin Inc., New York, 1966).

[7] S. W. Lovesey, Theory of Neutron Scattering from Condensed Matter (Oxford University Press, Oxford, 1986), Vol. 1.

[8] N. H. March, Liquid Metals (Cambridge University Press, Cambridge, England, 1990).

[9] U. Balucani and M. Zoppi, Dynamics of the Liquid State (Clarendon Press, Oxford, 1994).

[10] L. E. Bove, F. Sacchetti, C. Petrillo, and B. Dorner, Phys. Rev. Lett. 85, 5352 (2000).

[11] E. Guarini, U. Bafile, F. Barocchi, A. De Francesco, E. Farhi, F. Formisano, A. Laloni, A. Orecchini, A. Polidori, M. Puglini, and F. Sacchetti, Phys. Rev. B 88, 104201 (2013).

[12] F. Sacchetti, J. B. Suck, C. Petrillo, and B. Dorner, Phys. Rev. E 69, 061203 (2004).

[13] E. Pontecorvo, M. Krisch, A. Cunsolo, G. Monaco, A. Mermet, R. Verbeni, F. Sette, and G. Ruocco, Phys. Rev. E 71, 011501 (2005).

[14] S. Hosokawa, M. Inui, Y. Kajihara, K. Matsuda, T. Ichitsubo, W. C. Pilgrim, H. Sinn, L. E. González, D. J. González, S. Tsutsui, and A. Q. R. Baron, Phys. Rev. Lett. 102, 105502 (2009).

[15] V. M. Giordano and G. Monaco, Phys. Rev. B 84, 052201 (2011).

[16] V. M. Giordano and G. Monaco, Proc. Natl. Acad. Sci. U.S.A. 107, 21985 (2010).

[17] L. E. González and D. J. González, Phys. Rev. B 77, 064202 (2008).

[18] C. Petrillo, F. Sacchetti, E. Guarini, L. E. Bove, and F. Demmel, Phys. Rev. B 84, 094206 (2011).

[19] W. B. Pearson, A Handbook of Lattice Spacings and Structures of Metals and Alloys (Pergamon Press, Oxford, 1958). 
[20] Z. Li and J. S. Tse, Phys. Rev. Lett. 85, 5130 (2000).

[21] S. Blairs, J. Colloid Interface Sci. 302, 312 (2006).

[22] D. Aisa et al., Physica (Amsterdam) 385-386B, 1092 (2006).

[23] A. Orecchini, W. C. Pilgrim, C. Petrillo, J. B. Suck, and F. Sacchetti, Condens. Matter Phys. 11, 19 (2008).

[24] A. Orecchini, A. Paciaroni, A. De Francesco, L. Sani, M. Marconi, A. Laloni, E. Guarini, F. Formisano, C. Petrillo, and F. Sacchetti, Meas. Sci. Technol. 19, 034026 (2008).

[25] M. Zanatta, A. Fontana, A. Orecchini, C. Petrillo, and F. Sacchetti, J. Phys. Chem. Lett. 4, 1143 (2013).

[26] D. N. Zubarev, Sov. Phys. Usp. 3, 320 (1960).

[27] D. S. Sivia, Data Analysis: A Bayesian Tutorial (Clarendon, Oxford, 2005).

[28] C. F. Richardson and N. W. Ashcroft, Phys. Rev. B 50, 8170 (1994).
[29] L. E. Bove, F. Sacchetti, C. Petrillo, B. Dorner, F. Formisano, and F. Barocchi, Phys. Rev. Lett. 87, 215504 (2001).

[30] R. E. DeWames, T. Wolfram, and G. W. Lehman, Phys. Rev. 138, A717 (1965).

[31] N. J. Chesser and J. D. Axe, Phys. Rev. B 9, 4060 (1974).

[32] G. Monaco and V. M. Giordano, Proc. Natl. Acad. Sci. U.S.A. 106, 3659 (2009).

[33] E. Cornicchi, F. Sebastiani, A. De Francesco, A. Orecchini, A. Paciaroni, C. Petrillo, and F. Sacchetti, J. Chem. Phys. 135, 025101 (2011).

[34] A. Paciaroni, A. Orecchini, M. Haertlein, M. Moulin, V. C. Nibali, A. De Francesco, C. Petrillo, and F. Sacchetti, J. Phys. Chem. B 116, 3861 (2012).

[35] Y. Waseda, The Structure of Non-Crystalline Materials, (McGraw-Hill, New York, 1980). 